\title{
On-chip continuously tunable optical delay line based on cascaded Mach-Zehnder interferometers
}

\author{
Daniele Melati ${ }^{1, *}$ and Andrea Melloni ${ }^{2}$ \\ ${ }^{1}$ National Research Council Canada, 1200 Montreal Rd., Ottawa, ON K1A OR6, Canada \\ ${ }^{2}$ Dipartimento di Elettronica, Informazione e Bioingegneria, Politecnico di Milano, 20133 Milano, Italy \\ *daniele.melati@nrc-cnrc.gc.ca
}

\begin{abstract}
We propose a novel integrated optical delay line based on cascaded Mach-Zehnder interferometers. It allows a continuous delay tuning, can achieve wideband operation and is experimentally demonstrated with a delay up to $125 \mathrm{ps}$.
\end{abstract}

OCIS codes: (130.3120) Integrated optics devices; (230.7408) Wavelength filtering devices; (060.5625) Radio frequency photonics

\section{Introduction}

The advantages of integrated optical delay lines have been demonstrated for a variety of applications [1]. Common implementations exploit ring resonators to achieve group delay tuning up to several hundreds of picoseconds $[2,3]$. Although resonant delay lines make possible to obtain large delays with compact devices, this generally comes at the expense of large losses and narrow bandwidth [4]. Coupled resonators can increase the operational bandwidth but generally require complex tuning strategies. On the contrary, non-resonant delay lines achieve generally a smaller delay tunability compared to resonant devices but guarantee a much larger bandwidth and a finer control of the tuning [5].

In this work we propose a non-resonant optical delay line based on Mach-Zehnder interferometers equipped with tunable couplers. The delay line can be realized exploiting either a single stage or two cascaded identical stages. In both cases, the group delay can be tuned in a continuous manner by properly varying the coupling ratio of the couplers, without affecting power transmission at the operative wavelength (at least in the ideal lossless case), preventing modulation of the amplitude of the optical signal due to the reconfiguration of the circuit. Exploiting the proposed control scheme, the use of two cascaded stages allow to increase both the delay tunability and the bandwidth of the device compared to a single-stage Mach-Zehnder delay line. A single-stage device is realized on an InP-based photonic integration platform and experimentally demonstrated with a group delay tunability up to $125 \mathrm{ps}$.

\section{Optical delay line based on cascaded Mach-Zehnder interferometers}

The sketch of the proposed delay line is shown in Fig. 1 for the more general case of a circuit with two cascaded Mach-Zehnder interferometers and three tunable couplers. The coupling ratios $\mathrm{K}_{1}, \mathrm{~K}_{2}$ and $\mathrm{K}_{3}$ can be continuously varied between 0 and 1 . Tunable couplers are realized in this case with three balanced Mach-Zehnder equipped with phase shifters but any other coupler design could be used. Both the two stages have an unbalance $\Delta$ L between the two branches and phase shifters allow to tune the operative frequency of the device. For a single stage circuit the design is similar and includes only the first two tunable couplers $\mathrm{K}_{1}$ and $\mathrm{K}_{2}$ and the first unbalancing section. In general the device may have two input ports and two output ports but in this work it is used as a single-input single-output circuit in bar state.

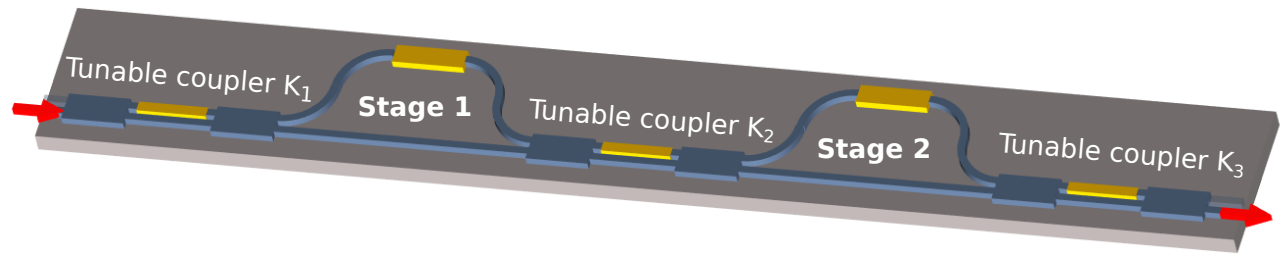

Fig. 1. Sketch of a two-stage optical delay line based on cascaded Mach-Zehnder interferometers. 


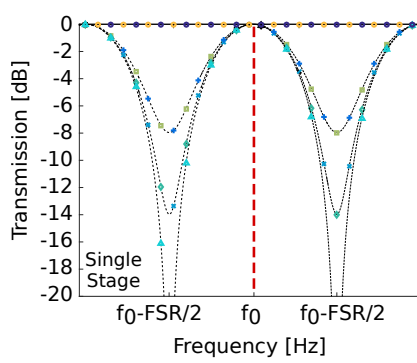

(a)

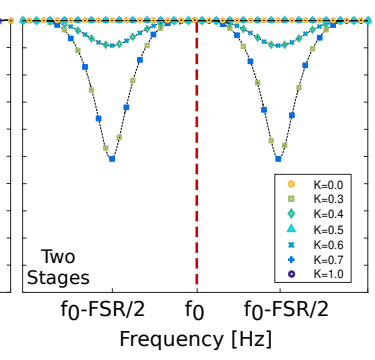

(b)

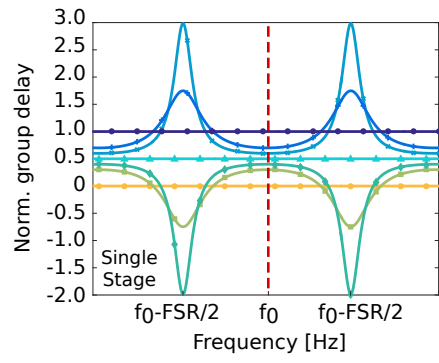

(c)

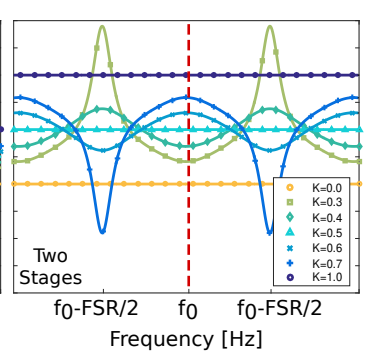

(d)

Fig. 2. Simulation of the normalized power transmission and group delay for $(\mathrm{a}, \mathrm{c})$ a single-stage delay line and $(b, d)$ a two-stage delay line.

First considering a single-stage design, we can define the operative frequency of the circuit $\mathrm{f}_{0}$ as the frequency such that $2 \pi f_{0} \Delta L / c+\phi=(2 N+1) \pi$, where $\phi$ is a constant phase shift applied through the phase shifter integrated in the interferometer [see Fig. 1] and $\mathrm{N}$ is an integer. If the two coupling ratios are chosen such that $\mathrm{K}_{1}=\mathrm{K}_{2}=\mathrm{K}$, the normalized group delay $\tilde{\tau}$ in bar state can be written as $\tilde{\tau}=\tau-\tau_{0} / T=\left(\tau-\tau_{0}\right) c /\left(n_{g} \Delta L\right)=K$, where $\tau_{0}$ is the minimum delay associated with the shortest branch of the interferometer, $\mathrm{n}_{g}$ is the group index of the waveguide and $\mathrm{c}$ is the speed of light. The minimum group delay $\tau_{0}$ corresponds to $\tilde{\tau}=0$ while $\tilde{\tau}=1$ corresponds to $\tau_{0}+\mathrm{T}$, the maximum group delay obtained travelling through the longest branch of the interferometer. The simulation of the power transmission of the circuit is reported in Fig. 2 (a) while Fig. 2 (c) shows the normalized group delay $\tilde{\tau}$, both represented as function of the light frequency for different values of $\mathrm{K}$ from 0 to 1 and neglecting propagation losses. As can be seen, at the frequency $\mathrm{f}_{0}$ (represented in Fig. 2 with red dashed lines) the intensity transfer function of the delay line is constant and does not depend on $\mathrm{K}$ while the normalized group delay increases linearly with $\mathrm{K}$. For the single-stage circuit the normalized 3-dB bandwidth around $\mathrm{f}_{0}$ can be calculated as $B=F S R / \pi \cdot \arccos \left[(K-0.5)^{2} / K(1-K)\right]$, with FSR the Free Spectral Range of the circuit. The minimum bandwidth of $B=F S R / 2$ is reached at $\mathrm{K}=0.5$.

The features of continuous delay tuning and constant output power at operative frequency can be retained also with a delay line realized with two cascaded identical Mach-Zehnder interferometers. In this case the three coupling ratios have to be chosen such that $\mathrm{K}=\mathrm{K}_{1}=\mathrm{K}_{3}=(\sin \theta)^{2}$ and $\mathrm{K}_{2}=(\sin 2 \theta)^{2}$, where $\theta$ is a proper control signal. For the design shown in Fig. 1, $\theta$ and $2 \theta$ are the phase delays induced by the phase shifter in the tunable couplers. This control strategy ensures the largest 3-dB bandwidth of the device and a linear dependence of the delay on K. Simulations of the power transmission and induced group delay of the circuit are shown in Fig. 2 (b) and (d), respectively, for different value of $\mathrm{K}$ (the choice of a value for $\mathrm{K}$ directly determines the coupling ratio of all the three couplers according to the relations reported above). As can be seen, the normalized group delay at the operative frequency now ranges from 0 to 2 , according to the linear relation $\tilde{\tau}=2\left(\tau-\tau_{0}\right) / T=2 K$. The actual group delay can hence be continuously changed from $\tau_{0}$ to $\tau_{0}+2 \mathrm{~T}$, doubling the tuning range of the delay line compared to a single-stage circuit. Interestingly this increase does not come at the expense of a narrower operative bandwidth, as can be seen in Fig. 2 (b). The minimum 3 - $\mathrm{dB}$ bandwidth is now obtained with $\mathrm{K}_{2}=0.5$ and is about $27 \%$ larger than that of a single Mach-Zehnder, resulting also in a more flat-top passband.

\section{Experimental results}

Single-stage optical delay lines were fabricated through a JePPIX Multi-Project Wafer Run on an InP-based technological platform [6]. Figure 3 (a) shows a photograph of one of the realized devices. Balanced 2x2 Mach-Zehnder interferometers are exploited as tunable couplers and realized with 3-dB MMI couplers and 250- $\mu \mathrm{m}$-long thermo-optic phase shifters. The same phase shifter is integrated also on one of the arm of the unbalanced Mach-Zehnder. The realized delay line has an unbalance $\Delta \mathrm{L}$ of $10.5 \mathrm{~mm}$ and an expected group delay variation for the TE polarized mode of about 123 ps. The experimental characterization of the devices was performed both in the optical domain (through an Optical Vector Analyser) and directly in the RF domain exploiting a Vector Network Analyser and an electro-optic modulator, obtaining similar results. The measured group delay at the operative wavelength $\lambda_{o}=1534.96 \mathrm{~nm}$ as function of the current $I_{c}$ fed into the two tunable couplers for TE polarized input light is shown in Fig. 3 (b). Solid line represents a fit performed on the experimental data with a squared cosine model. Minimum delay is achieved with a $\mathrm{I}_{\mathrm{c}}$ $\simeq 24 \mathrm{~mA}(\mathrm{~K} \simeq 0)$, causing light to travel in the shortest branch of the Mach-Zehnder interferometer. On the contrary, light travels only in the longest branch of the delay line (maximum delay) for $\mathrm{I}_{\mathrm{c}} \simeq 78 \mathrm{~mA}(\mathrm{~K} \simeq 1$ ). The measured variation of the group delay at the operative wavelength is about $124 \mathrm{ps}$. The measured normalized power transmission 

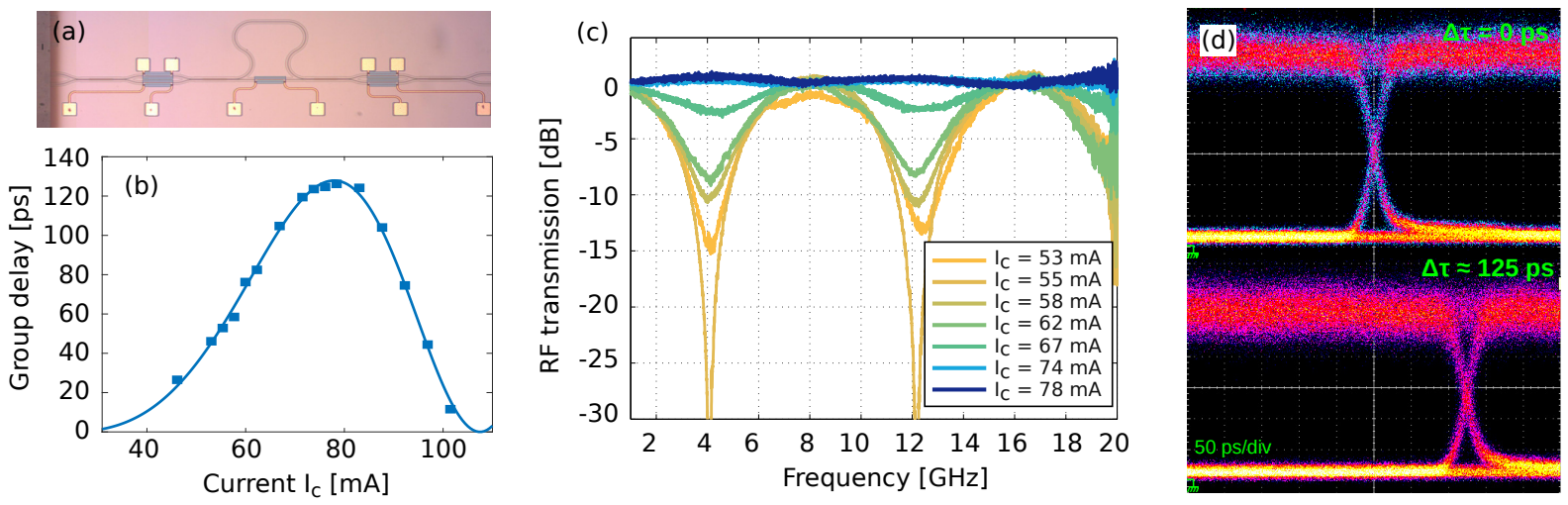

Fig. 3. (a) Photograph of the realized single-stage delay line; (b) measured group delay variation as function of the current $I_{c}$ fed into the controllers of the tunable couplers; (c) normalized transmission as function of the frequency for different values of the tuning current $\mathrm{I}_{c}$. (d) Eye diagrams of a $1.25 \mathrm{Gbit} / \mathrm{s}$ OOK NRZ signal propagating through the circuit with different group delay settings.

spectrum is shown in Fig. 3 (c) for several values of $I_{c}$ as function of the frequency of the RF signal modulated on the optical carrier at $\lambda_{o}=1534.96 \mathrm{~nm}$. The measured spectrum is in good agreement with the simulations shown in Fig. 2 (a). Increasing $I_{c}$ a strong frequency dependence appears in the spectrum. As expected the deepest notches are obtained with $\mathrm{I}_{\mathrm{c}}=55 \mathrm{~mA}(\mathrm{~K} \simeq 0.5)$ while for $\mathrm{I}_{\mathrm{c}}=78 \mathrm{~mA}(\mathrm{~K} \simeq 1)$ power transmission is again wavelength independent. The measured FSR of about $8 \mathrm{GHz}$ is consistent with the maximum measured group delay variation of $124 \mathrm{ps}$. At $\mathrm{I}_{\mathrm{c}}=55$ $\mathrm{mA}$, the $3-\mathrm{dB}$ bandwidth is about $4.45 \mathrm{GHz}$. The obtained results were verified by transmitting a $1.25 \mathrm{Gbit} / \mathrm{s} \mathrm{OOK}$ NRZ signal through the integrated delay line and recording the eye diagrams for different values of $I_{c}$. Figure 3 (d) shows the results for $I_{c}=0$ (minimum delay) and $I_{c}=78 \mathrm{~mA}$ (delay increased of about $124 \mathrm{ps)}$. As can be seen the delay of the signal can be changed up to about $125 \mathrm{ps,} \mathrm{confirming} \mathrm{the} \mathrm{functionality} \mathrm{of} \mathrm{the} \mathrm{proposed} \mathrm{delay} \mathrm{line.}$

\section{Conclusions}

We have reported on the design of an integrated non-resonant optical delay line based on cascaded Mach-Zehnder interferometers. A continuous control of the delay is achieved by varying the coupling ratio of the integrated tunable couplers, without affecting the power transmission at the operative frequency. Using two cascaded stages and controlling the tunable couplers according to the proposed scheme allows to double the delay tunability and increase the bandwidth of the device of about $27 \%$ compared to a single-stage Mach-Zehnder. The functionality of a device with a single stage was experimentally demonstrated with a group delay tunability up to about 125 ps.

\section{References}

1. A. Meijerink, C. G. Roeloffzen, R. Meijerink, L. Zhuang, D. A. Marpaung, M. J. Bentum, M. Burla, J. Verpoorte, P. Jorna, A. Hulzinga et al., "Novel ring resonator-based integrated photonic beamformer for broadband phased array receive antennas-Part I: Design and performance analysis," Journal of Lightwave Technology 28, 3-18 (2010).

2. A. Canciamilla, M. Torregiani, C. Ferrari, F. Morichetti, R. De La Rue, A. Samarelli, M. Sorel, and A. Melloni, "Silicon coupled-ring resonator structures for slow light applications: potential, impairments and ultimate limits," Journal of Optics 12, 104,008 (2010).

3. J. B. Khurgin and P. A. Morton, "Tunable wideband optical delay line based on balanced coupled resonator structures," Optics letters 34, 2655-2657 (2009).

4. G. Lenz, B. Eggleton, C. K. Madsen, and R. Slusher, “Optical delay lines based on optical filters,” IEEE Journal of Quantum Electronics 37, 525-532 (2001).

5. V. C. Duarte, M. V. Drummond, and R. N. Nogueira, "Photonic true-time-delay beamformer for a phased array antenna receiver based on self-heterodyne detection," J. Lightwave Technol. 34, 5566-5575 (2016).

6. D. Melati, F. Morichetti, A. Canciamilla, D. Roncelli, F. Soares, A. Bakker, and A. Melloni, "Validation of the building-block-based approach for the design of photonic integrated circuits," Journal of Lightwave Technology 30, 3610-3616 (2012). 